\title{
ASPECTOS DE LA COMUNICACIÓN INTERNA EN INSTITUCIONES DE EDUCACIÓN SUPERIOR
}

\section{ASPECTS OF INTERNAL COMMUNICATION IN INSTITUTIONS OF HIGHER EDUCATION}

\author{
Mendoza-Zamora Walter ${ }^{1 *}$; Hormaza-Muñoz Zaida²; García-Arauz Jahaira³; Chamba- \\ Rueda Laura ${ }^{4}$ \\ ${ }^{1}$ Docente de la Universidad Estatal del Sur de Manabí, UNESUM. Jipijapa, Ecuador. \\ ${ }^{2,3}$ Docente de la Universidad Laica Eloy Alfaro de Manabí, ULEAM. Manta, Ecuador. \\ ${ }^{2}$ Docente de la Universidad Técnica Particular de Loja, UTPL. Loja, Ecuador.
}

*Correo: wal-mezam82@gmail.com

\begin{abstract}
Resumen
La comunicación se define como una oportunidad de encuentro y de intercambio de información con los demás, esta plantea una amplia gama de posibilidades para la interacción social, ya que a través de ella se puede lograr el entendimiento, la coordinación y la colaboración entre los diferentes actores dentro de una organización para su desarrollo y crecimiento. La presente investigación tiene como objetivo revisar la conceptualización de los aspectos que rigen el proceso de comunicación interna en las universidades. De modo que la comunicación universitaria asiste de nuevo a las fuentes doctrinales de las que tradicionalmente ha gustado. Sobre todo, la comunicación organizacional, que a lo largo del último medio siglo ha logrado atesorar un conjunto de saberes que han garantizado el eficaz desempeño de esa actividad profesional. se pudo conocer que los medios de comunicación se han convertido en ámbitos privilegiados de espacios públicos para brindar información, propaganda, publicidad y consenso, con el propósito de expandir una cultura corporativa.
\end{abstract}

Palabras clave: comunicación institucional, plan de comunicación, medios de comunicación

\begin{abstract}
Communication is defined as an opportunity for meeting and exchanging information with others, this poses a wide range of possibilities for social interaction, since through it you can achieve understanding, coordination and collaboration between different actors within an organization for its development and growth. This research aims to review the conceptualization of the aspects that govern the internal communication process in universities. Thus, university communication once again assists the doctrinal sources that it has traditionally liked. Above all, organizational communication, which over the last half century has managed to treasure a set of knowledge that has guaranteed the effective performance of that professional activity. It was learned that the media have become privileged areas of public spaces to provide information, propaganda, advertising and consensus, with the purpose of expanding a corporate culture.
\end{abstract}

Keywords: institutional communication, communication plan, media.

Información del manuscrito:

Fecha de recepción: 02 de agosto de 2017

Fecha de aceptación: 06 de diciembre de 2017

Fecha de publicación: 10 de enero de 2018 


\section{Introducción}

La comunicación se entiende como una oportunidad de encuentro y de intercambio de información con los demás, esta plantea una amplia gama de posibilidades para la interacción social, ya que a través de ella se puede lograr el entendimiento, la coordinación y la colaboración entre los diferentes actores dentro de una organización para su desarrollo y crecimiento. En lo que respecta a la comunicación integral, esta se inicia en el siglo XIX, en un tiempo de cambios y agitación promovido por la revolución industrial, pero su definición asertiva fue propuesta por Joan Costa en la década de los 70 , con la cual se ha revolucionado la visión de pensar y trabajar comunicativamente, tomando en consideración la forma de ser planificada y gestionada, modificando sustancialmente la forma de abordar cualquier proyecto de comunicación (Arrugo, 2001; Llanura, 2010).

La carencia de la toma de decisiones en las instituciones de educación superior por la falta de habilidades para la comunicación, acaban neutralizando las acciones $y$ actividades que se deben realizar, ya que aparecen como incapaces de sumar sus esfuerzos en un sentido estratégico que permita alcanzar la calidad a base de unas competencias requeridas por la sociedad y que estén vinculadas a las nuevas tecnologías 0 a los sistemas de información que en la actualidad tienen un componente comunicativo esencial para el desarrollo humano (Sánchez, 2005).

En relación a las universidades, progresivamente, cada una de estas han ido ampliando su actividad hacia la comunicación interna, donde necesariamente se tiene que dar una relación con el entorno a través del marketing, publicidad otro tipo de comunicación directa o indirecta (Sánchez, 2005; Ballester \& Ramos, 2009).

Por lo tanto, la presente investigación tiene como objetivo revisar la conceptualización de los aspectos que rigen el proceso de comunicación interna en las universidades.

\section{Comunicación}

Arrugo (2001) describe que la comunicación es el conjunto de herramientas que como seres humanos se usan para interpretar, 
reproducir, mantener y transformar el significado de las cosas y de esta forma estar en comunicación dentro de la cultura que le corresponde. La comunicación, entonces, constituye la esencia de la cultura, de la empresa, de la vida misma (p. 9).

Establecer cualquier proceso de comunicación implica comunicar un determinado contenido a otras personas 0 desempeñar muchas otras funciones. Llanura (2010) menciona que: "A la comunicación se la considera un proceso mediante el cual las personas interaccionan entre sí, de acuerdo a símbolos que representan información, ideas, actitudes y emociones con el propósito de influirse de forma mutua" (p. 3).

La comunicación es un fenómeno de carácter social, en ella se integran todos los actos con los cuales los individuos intercambian información. Sin embargo, muchas organizaciones tienen problemas para plantear y gestionar integralmente su comunicación, ya que no saben por dónde empezar. Es frecuente tener la sensación que desde el equipo de la dirección están herrados y sus políticas no se comprenden porque no son bien explicadas o no cumplen con los requerimientos que la comunidad necesita, razón por la cual existen problemas de comunicación en las instituciones.

Analizando la obra de Westpalen (2014), se pudo conocer que: el concepto de comunicación integral que define la actitud ante la comunicación en las organizaciones contemporáneas incluye todas las formas de comunicación y es aplicable a todas las organizaciones, independientemente de la actividad que desarrollen. Integral quiere decir que se acepta como planteamiento básico que todo comunica y que, por tanto, toda la comunicación debe gestionarse globalmente desde un único departamento. Es un cambio no sólo de concepto, sino que afecta a la estructura de la organización porque supone superar el modelo organizativo en el que cada departamento gestiona su comunicación y la comunicación interna se asocia al área de recursos humanos como una forma más de relación o control sobre los empleados con los que se relaciona. La dirección de recursos humanos conoce mejor los instrumentos que orquestan la política de personal, 
pero, por otra parte, como muestra la evidencia de muchas empresas, corre el riesgo de posponer los temas de comunicación ante otras prioridades que, día a día, le parecen más conflictivas e importantes ( $p$. 351).

Cabe destacar que una gestión adecuada de la comunicación interna en las organizaciones, ocupa un lugar esencial en los planes estratégicos, para asegurar una correcta implantación de las metas y objetivos que se planteen. La comunicación favorece y promueve las relaciones eficientes entre los diferentes actores organizacionales.

La gestión de comunicación interna en las instituciones, se ha convertido en una tarea compleja, considerando que en este contexto conviven grupos sociológicamente diversos, cada uno con un conocimiento y enfoque que lo hacen diferente, con demandas y expectativas diferentes, que en conjunto y con conciencia de desarrollo, logran sacar adelante los propósitos socioeducativos por los que fueron creados, entendida desde la percepción de sus públicos, así como del uso que se hace de los diferentes canales y herramientas que están en funcionamiento para comunicarse y que pueden ser físicas y/o digitales.

\subsection{Medios de comunicación}

Los medios de comunicación permiten generar todo tipo de acto y acción social, por ello están considerados de forma amplia, y pueden ser: prensa, teléfono móvil y celular, palabra escrita, palabra impresa, carteleras, avisos, periódico institucional, correos electrónicos, intranet (correo institucional), internet, radio, televisión, entre otros. Por ello, todos los actos humanos están involucrados en algún tipo de medio para comunicarnos. Según West, (2005): "Los medios de comunicación inspiran todo acto y acción de la sociedad. Los medios de comunicación fijan nuestras percepciones y organizan nuestras experiencias. Los medios de comunicación ponen el mundo en contacto" (p. 383).

Es decir, los medios de comunicación ponen el mundo en contacto con la sociedad, sus efectos pueden ser positivos 0 negativos, tanto en la parte política, económica, social, familiar y cultural. Marc (2009) describe que: "Los medios de comunicación, tienen una 
gran capacidad para proveer de recursos simbólicos y herramientas con el fin de comprender la complejidad de la vida cotidiana" ( $p$. 15).

Los medios de información, tienen como misión estratégica la información sobre la información, que se da a través de la inteligencia, interpretación, filtrado y búsqueda efectiva de datos que influencien de forma positiva $y / 0$ negativa el desarrollo social, laboral y familiar. Cabe considerar que en la actualidad son los medios de información tecnológicos los que están en su mayor auge por su forma rápida para informar. Briggs (2007) describe que: "la nueva tecnología y los dispositivos, siempre en evolución, han cambiado y continuarán cambiando- los mercados que las operaciones periodísticas están apuntando a servir" (p. 42).

\section{Líneas de acción para la comunicación universitaria}

En la época actual, existe la necesidad de adaptarse a los cambios de la revolución industrial, tecnológica y por ende la comunicacional, mismas que se dan también en el campo universitario, donde se observa cómo éstos suceden con rapidez, sobre todo, entre algunos de sus stakeholders, es decir, de los administradores, clientes, grupos responsables de la entidad como los directivos, comunidad y sociedad en general. Por ello, se requiere de una política de comunicación para su desarrollo (Drucker, 1969).

Lo expuesto, permite reflexionar, que la comunicación universitaria, debe continuar superando el fuerte peso de una tradición cultural en lo tocante al ejercicio de la comunicación para dar paso al modelo basado en la emisión de mensajes unidireccionales, mismas que van variando por el incremento en el volumen de informaciones que acumulaban y por establecerse un nuevo modelo de relaciones con los administradores hacia los que deben tener mayor transparencia para alcanzar el desarrollo deseado.

De modo que la comunicación universitaria asiste de nuevo a las fuentes doctrinales de las que tradicionalmente ha gustado. Sobre todo, la comunicación organizacional, que a lo largo del último medio siglo ha logrado atesorar un conjunto de saberes que han garantizado el eficaz 
desempeño de esa actividad profesional. $Y$ es que esa comunicación especializada ha resultado útil; incluso necesaria, para la sociedad en cuyo seno se ha desplegado, adecuándose a los modelos empresariales imperantes y a los modelos comunicativos consecuentes. Al atesorar ese cúmulo de saberes, al mismo tiempo se normaliza dicha actividad profesional, es decir, la comunicación organizacional ha logrado alcanzar un notable proceso en las instituciones, conquistando en los actuales momentos un espacio profesional propio; que entre otras cosas, incluye el acopio de cierto saber hacer, la fijación de determinados roles profesionales y el establecimiento de un soporte institucional.

\subsection{Comunicación interna}

La comunicación es considerada como una herramienta clave para dar respuesta a los diferentes cambios generados en la actualidad $y$ a los que debe someterse una empresa, misma que ha sido creada para brindar un servicio y producir beneficios. En esta época de cambios, la comunicación obviamente, no podía quedarse al margen de ellos. Por el contrario, este ámbito de la actividad humana, que tanto protagonismo alcanza en la sociedad contemporánea, conoce ahora muchas formas para comunicarse.

Con términos de ese estilo aluden a novedades que tienen sobre todo que ver con las máquinas de comunicar y con los usos que se les da a los escenarios de la vida cotidiana; donde se personalizan las actividades comunicativas y se despliega con cierta frecuencia algunas prácticas culturales de nuevo modelo. Por lo tanto, se entiende a la comunicación como una oportunidad de encuentro y de intercambio de información con los demás, esta plantea una amplia gama de posibilidades para la interacción social, ya que a través de ella se puede lograr el entendimiento, la coordinación y la colaboración entre los diferentes actores dentro de una organización para su desarrollo y crecimiento. Ballester \& Ramos (2009) en su texto describe que: la comunicación interna se enmarca en las acciones generales de comunicación, pero no siempre es comunicación corporativa; es decir, no siempre la 
comunicación es global de la organización como totalidad, sino que en ocasiones responde a prioridades y objetivos de departamentos concretos (pp. 8586).

Sánchez (2005) menciona que: "la comunicación interna en las universidades tiene que dar a conocer y hacer comprender la situación de la organización, lograr el compromiso de todos sus miembros y facilitar la participación" (p. 165). Así mismo Cuena-Boltrán (2005) insiste que el objetivo final de la comunicación interna en la universidad es: que la comunidad universitaria conozca lo que hace la institución y cuáles son sus proyectos y resultados; persigue lograr un clima de implicación e integración entre los miembros e incrementar la motivación y la participación. En buena medida, soporte y base de la comunicación externa (p. 114).

Ballester \& Ramos (2009) en su texto describe que: comunicar internamente es comunicar puertas adentro, en todas las direcciones. No es que se establezca un único flujo de información, ni que todos los miembros compartan el mismo mensaje, esto es que la comunicación interna es una acción multidireccional y transversal, que tiene como destinatarios o como emisores a cualquiera de los miembros de la organización. Es multidireccional, porque se encarga tanto de los avisos de la dirección al personal como de las notas y reclamaciones que el personal deja en el buzón de sugerencias, por ejemplo; y es transversal, porque puede involucrar a varios departamentos (pp. 85-86).

En relación a las universidades, progresivamente, cada una de estas han ido ampliando su actividad hacia la comunicación interna, donde necesariamente se tiene que dar una relación con el entorno a través del marketing, publicidad otro tipo de comunicación directa o indirecta. Según Túnez (2012) necesario conocer los diferentes tipos de comunicación social:

Los intermediarios. Masiva e interpersonal.- Masiva, la que se establece cuando el proceso de comunicación tiene como receptor a grupos numerosos de individuos. Interpersonal. La comunicación interna es la comunicación entre actores, independientemente de su 
ubicación en el organigrama, y muchas veces esa comunicación se hace de persona a persona. La comunicación interpersonal en las organizaciones está presente en las reuniones de los equipos directivos, de trabajo entre varios departamentos, en la transmisión de mensajes. El canal de difusión: mediada y directa. - Mediada, la que se establece a través de un soporte de difusión del mensaje. Directa, cuando el contacto entre emisor y receptor es personal (p. 80).

Esto es, a reconocer que las instituciones, no pueden dejar de comunicarse de forma interna 0 externa, misma que no se comunica solo con palabras o símbolos, sino también mediante sus comportamientos y muchos otros elementos que demuestran sus acciones y respuestas que la comunidad y sociedad requiere. $Y$ es que la comunicación recorre transversalmente y de manera continua, aunque adoptando diversas formas para concebir $y$ orientar correctamente sus actuaciones comunicativas. Para ello requiere de acciones que le ayuden a comunicar de forma efectiva. Según Robbing \& Judge (2009), los medios para la comunicación las ordenan, de menos a más, según su efectividad haciendo uso de: "reportes formales y boletines, memorandos y cartas; discursos grabados; correo electrónico; grupos de discusión en línea; correo de voz; discursos en vivo; conversaciones telefónicas; videoconferencias; y conversaciones cara a cara" (p. 367).

La comunicación, entonces, ha ido ganando posiciones a lo largo de la historia universitaria. Ello obedece, por supuesto, a diferentes razones, factores, condiciones, agentes y acciones, que han sido generadores de un cambio social. Todo ello, ha permitido que la universidad evolucione, dando cumplimiento a los fines y las funciones que a cada uno de sus miembros le competen; en última instancia, al modelo de comunicación que la universidad de acuerdo al momento establezca. Es importante entonces, tener una iniciativa para comunicarse.

\section{Conclusiones}

se realizó la revisión bibliográfica para analizar diversas teorías, con lo cual se pudo conocer que los medios de comunicación se han convertido en ámbitos privilegiados de espacios públicos para brindar información, 
propaganda, publicidad y consenso, con el propósito de expandir una cultura corporativa. Esto indica que una adecuada comunicación, se la llevará a través de la dirección de empresas y el papel que juega la comunicación interna en las diferentes tipologías de la organización, según sus niveles de estructuración y las relaciones entre sus públicos.

\section{Bibliografía}

Arrugo, M. (2001). El fin de la comunicación interna. A Trabajar. Recuperado el 19 de agosto de 2015, de http://www.atrabajarpr.com

Ballester, C., \& Ramos, I. (2009). Las relaciones públicas en la gestión de la comunicación interna. Recuperado el 17 de enero de 2015, de http://rua.ua.es/dspace/bitstre am/10045/15719/1/LIBRO\%2 OLas\%20relaciones\%20p\%C $3 \%$ BAblicas\%20en\%20la\%20 gesti\%C3\%B3n\%20de\%20la \%20comunicaci\%C3\%B3n\%2 Ointerna.pdf

Briggs, M. (2007). Periodismo 2.0. Una guía de alfabetización digital. Ed. Jan Schaffer. Trad. Guillermo Franco. Knight Foundation.

Cuena-Botrán, P. (2005). La dirección de comunicación en el ámbito universitario: comunicación interna y comunicación externa". En Marín Ruiz, A.; Trilles Rodriguez, I. y Zamarrón Garza, G. (coods.), Mass Media y Universidad. El reto de la comunicación.

Drucker, P. (1969). The age of discontinuity. Londres: Willians Heinemann Lid.

Llanura, P. (2010). La comunicación en las organizaciones. Ministerio de trabajo y asuntos sociales España. Recuperado el 13 de enero de 2015, Ministerio de trabajo y asuntos sociales España. Disponible en:

http://www.insht.es/InshtWeb/ Contenidos/Documentacion/F ichasTecnicas/NTP/Fiche

Marc, S. (2009). Políticas de Educación en medios. Aportaciones y Desafíos mundiales. Prólogo. En "Comunicar. Revista Científica Iberoamericana de Comunicación y Educación", XVI, 32. Marzo, 2009. Número especial coeditado con la Alianza de civilización.

Robbing, P., \& Judge, T. (2009). Comportamiento organizacional. México, Distrito Federal: Pearsons. Decimotercera edición.

Sánchez, M. (2005). Comunicación interna en la universidad: el reto de conseguir la participación de los universitarios. En De Salas, I.; Fernández, A.B. y Martínez, 
F., La universidad en la comunicación, la comunicación en la universidad.

Túnez, M. (2012). La gestión de la comunicación en las organizaciones. $1^{\text {a }}$ edición, Manganeses de la
Lampreana. España: Editorial Comunicación Social S. C.

West, R. T. (2005.). Teoría de la Comunicación. Análisis y aplicación. Madrid: Editorial, McGraw-Hill.

Westpalen,

$\mathrm{H}$.

(2014). 